\title{
Salud visual y percepción social de malabaristas de la ciudad de Bogotá, Colombia
}

\author{
Visual health and social perception of jugglers in the city of Bogotá, Colombia
}

Kelly Nataly Rincón-Suarez' ${ }^{1}$ orcid.org/0000-0003-3719-2459

Ingrid Astrid Jiménez-Barbosa ${ }^{* *}$ orcid.org/0000-0001-7874-2598

1 Universidad de la Salle. Bogotá, Colombia

Fecha de recepción: Enero 19 - $2017 \quad$ Fecha de revisión: Septiembre 21 - $2017 \quad$ Fecha de aceptación: Diciembre 1 - 2017

Rincón-Suárez KN, Jiménez-Barbosa IA. Salud visual y percepción social de malabaristas de la ciudad de Bogotá, Colombia. Univ. Salud. 2017;19(3):340-351. DOI: http://dx.doi.org/10.22267/rus.171903.96

\section{Resumen}

Introducción: El malabarismo es una práctica artística que comprende diversas dinámicas históricas, sociales, culturales, económicas y en la actualidad concibe nuevos enfoques con relación la salud del artista. Objetivo: Comprender el estado de la salud visual y la percepción social del malabarismo en la ciudad de Bogotá. Materiales y métodos: Se realizó un estudio mixto combinando metodología cualitativa tipo fenomenológica y cuantitativa descriptiva en un grupo de 15 malabaristas de la ciudad de Bogotá que laboraban en los semáforos de las localidades de Teusaquillo, Suba y Engativá, por medio de tres etapas: Planeación; Trabajo de Campo incluye diario de campo, entrevista y pruebas de tamizaje visual y ocular; Campaña publicitaria. Resultados: El 100\% de los participantes refirió enrojecimiento ocular y sensación de arenilla que se asocia con las horas de trabajo, los instrumentos de manipulación y la exposición al ambiente laboral. Hay normalidad en la percepción de la profundidad (20" de arco) y la percepción al color en la totalidad de participantes. Conclusiones: La salud visual y ocular no tiene gran importancia para los malabaristas participantes, sin embargo presentan síntomas de molestia ocular. El malabarista se percibe como un actor social que contribuye con el crecimiento de la ciudad sin importar que el común de la gente considere esta práctica no saludable. La campaña social diseñada logró sensibilizar a transeúntes y conductores.

Palabras clave: Percepción social; percepción visual; salud ocular; desempeño psicomotor; sector informal. (Fuente: DeCS, Bireme).

\begin{abstract}
Introduction: Juggling is an artistic practice that includes various historical, social, cultural and economic dynamics, and nowadays it conceives new approaches regarding the artist's health. Objective: To understand the state of visual health and the social perception of juggling in the city of Bogotá. Materials and methods: A mixed study combining qualitative methodology of phenomenological type a quantitative descriptive methodology in a group of 15 jugglers of the city of Bogota, who worked at the traffic lights in the localities of Teusaquillo, Suba and Engativá, through three stages: planning; field work which includes field diary, interview and visual and ocular screening tests; and advertising campaign. Results: $100 \%$ of the participants referred to eye reddening and feeling of grit that is associated with the working hours, manipulation tools and exposure to the work environment. There are normally in the perception of depth (20" of arc) and the perception of color in the totality of participants. Conclusions: Visual and ocular health is not very important for the participating jugglers; however, they have symptoms of ocular discomfort. The juggler is perceived as a social actor that contributes to the growth of the city no matter that the common people consider this practice as unhealthy. The designed social campaign managed to sensitize pedestrians and drivers.
\end{abstract}

Keywords: Social perception; visual perception; ocular health; psychomotor performance; informal sector (Source: DeCS, Bireme). 


\section{Introducción}

El malabarismo es una práctica artística escénica que se agrupa en las diversas técnicas creativas desde tiempos pasados (1930) haciendo parte del crecimiento de diferentes generaciones a nivel global, como se observa en la representación de películas y $\operatorname{circos}^{(1,2)}$.

Hoy en día, el fenómeno del malabarismo se toma las calles de la ciudad como una actividad artística independiente, que contribuye con la formación de creencias y costumbres en la sociedad. A su vez esta actividad ha permitido que el malabarista pueda cubrir sus necesidades invadiendo espacios cotidianos abiertos, como lo son los semáforos convirtiéndose en parte de esta cultura conocida como "la sociedad del semáforo"(3) o "la cultura del rebusque" e informalidad(4). No obstante, hoy en día es común asociar esta actividad con estigmas sociales como por ejemplo que quienes lo practican son drogadictos o alcohólicos, sin pensar que gracias a sus habilidades tienen la posibilidad de ganar recursos económicos(5).

En la ciudad de Bogotá el $27 \%$ de artistas urbanos practican el malabarismo sin importar su estrato social, lo que indica que algunos cuentan con acceso a seguridad social en salud(6), pero respecto a lo laboral aún no cuentan con un servicio que los cubra ante una enfermedad o accidente. Hay que tener presente que este trabajo involucra exposición diaria a diferentes riesgos como sustancias químicas cuando emplean fuego, vapores y gases procedentes de los vehículos; físicos como la exposición a la luz solar y por ende a la radiación ultravioleta; ergonómicos como posturas habituales incorrectas, trabajo repetitivo (contenido de la tarea), frente a la manipulación de diferentes instrumentos como los diábolos, clavas, antorchas o pelotas en un espacio variable e indefinido(7). Por otro lado, su salud en general y específicamente la visual y ocular pueden verse afectadas diariamente. Hay que recordar que el desarrollo de la visión espacial y de profundidad estarán acompañados por una serie de cambios anatómicos y fisiológicos que se desarrollan desde el nacimiento hasta la estimulación lograda por la experiencia, por lo cual el inadecuado desarrollo en los ojos podría generar alteraciones en el desarrollo de la actividad del malabarismo, así como la percepción al color desde el punto de vista del desarrollo activo de su trabajo. En cuanto a la salud ocular, la principal sintomatología esperada se relaciona con problemas de sequedad y fatiga ocular asociada a las horas de trabajo al aire libre que se encuentra contaminado(8).

De acuerdo con lo anotado anteriormente la presente investigación se propuso comprender el estado de la salud visual y la percepción social del malabarismo en la ciudad de Bogotá, con base en la cual se desarrolló una campaña social para dar a conocer a los actores sociales (conductores y transeúntes) los resultados obtenidos en el estudio. Esta investigación aporta información actualizada sobre este fenómeno urbano, que tiene un abordaje escaso dando así bases de referencia para futuros estudios e investigaciones con un enfoque mixto en relación a la salud y temas sociales.

\section{Materiales y métodos}

\section{Diseño del estudio}

Se realizó un estudio mixto el cual combinó la metodología cualitativa tipo fenomenológica y cuantitativa descriptiva( ${ }^{(9)}$. Se incluyeron quince malabaristas con edades entre 19 a 25 años en la ciudad de Bogotá que laboran en los semáforos de las localidades de Teusaquillo, Suba y Engativá. La selección de esta muestra se realizó por conveniencia utilizando la estrategia de bola de nieve. Para el tamaño de muestra de la parte cuantitativa, se tuvo en cuenta una potencia de $80 \%$ y un error tipo I del $5 \%$ basado en los reportes sobre número de malabaristas que existen en las localidades mencionadas. Una vez seleccionados los participantes de la investigación, se determinaron las etapas para el desarrollo de la misma, las cuales son: planeación, trabajo de campo y campaña publicitaria.

\section{Etapa de planeación}

El grupo investigador contó con un nombre y una filosofía de trabajo para ser identificados. Se 
revisaron los antecedentes de la trayectoria del malabarismo en el mundo desde sus orígenes y cómo ésta práctica ha sido investigada desde el punto de vista cultural, antropológico y social en la ciudad de Bogotá. Con esta base documental, se pudo establecer que el malabarismo ha sido estudiado por parte del Instituto Distrital de Patrimonio Cultural y por estudiantes de Facultades del área de las ciencias sociales, considerando principalmente variables socioeconómicas en términos de ingresos y formas de subsistencia, más no desde la propia percepción del malabarista frente a la inclusión en la sociedad, el punto de vista desde el desarrollo de su arte respecto a su nivel social y mucho menos sobre la importancia de la salud visual y ocular al desempeñar su trabajo. Por lo tanto, el grupo, habiendo identificado la problemática estableció una serie de actividades orientadas a la comprensión del fenómeno a estudiar(1,9).

\section{Etapa de trabajo de campo}

Inicialmente se realizó observación participante como técnica de recolección de información directa o interactiva. Esta buscó compartir de manera descriptiva el contexto, experiencias y vida cotidiana, de cada uno de los sujetos estudiados. En un diario de campo se describió de manera detallada el lugar de trabajo (semáforo), interacción con actores sociales (transeúntes y conductores), lugar de práctica, identificando posibles riesgos para la salud general $\mathrm{y}$ visual. Para interactuar con los participantes del estudio, se realizó una entrevista de tipo grupal para proporcionar un control de calidad sobre los datos obtenidos. Se validó esta entrevista previamente realizando una prueba con un grupo de 30 personas que contaban con características similares respecto a su nivel educativo, estrato social y edad.

Los quince malabaristas participantes respondieron a cada una de las preguntas dentro del grupo focal, permitiendo conocer si la respuesta era aprobada o suprimida por los otros entrevistados(10). Esta herramienta permitió conocer las costumbres, normas internas y la visión acerca de si mismos. Las entrevistas fueron grabadas en video, así como un día de trabajo en el semáforo y sus días de práctica previos.

Para el posterior análisis, las entrevistas fueron transcritas en archivo Word. Se realizó una codificación de primer nivel, en la cual emergieron ideas para realizar un análisis descriptivo por medio de categorías y subcategorías. Una vez identificadas estas categorías se procedió a realizar una codificación de segundo nivel en la cual se realizaron comparaciones entre dichas categorías y se realizó la interpretación y explicación de la hipótesis de trabajo con éstas.

Posteriormente, se aplicó el cuestionario clínico de Donate, validado internacionalmente para indagar la intensidad y frecuencia de los síntomas oculares en pacientes que tienen sospecha de ojo seco(11). En este caso, se utilizó para evaluar la sintomatología ocular presentada por cada uno de los malabaristas (Tabla 1), considerando la sospecha de ojo seco o molestia visual cuando se reportan puntajes iguales o superiores a 13. Para evaluar aspectos de la función visual importantes en este tipo de trabajo, se aplicó la prueba de tamizaje Random Dot que permite conocer si existe o no una buena visión de profundidad (3D o estereopsis) (Figura 1), para que el desempeño realizado en los malabares sea de precisión y calidad. El valor considerado como normal fue de $20^{\prime \prime}$ de arco(12).

Para evaluar la visión al color, se aplicó la prueba de Ishihara, que permite establecer si hay normalidad o no en la visión cromática(13). Estas pruebas no requieren ser aplicadas en condiciones especiales de consultorio, no obstante, el lugar escogido debe tener buenas condiciones de iluminación (Figura 2). Dentro de las causas más frecuentes de ojo seco se encuentran las largas horas de exposición al sol, encontrarse en ambientes secos o con exceso de viento factores que incluyen la participación de los pacientes de este estudio. A su vez, la percepción en profundidad es la función del sistema visual que permite distinguir con alta precisión la tridimensionalidad, por lo cual para el malabarista es una función primordial ya que en el lanzamiento y recogida de bolas y clavas 
involucra la coordinación y destreza sensomotora, lo que indica que debe existir una serie de elementos que permitan un buen funcionamiento binocular que logre una adecuada interpretación de la imagen, más si esta se encuentra en movimiento, lo que implica una adecuada coordinación óculo-manual. Por otro lado, la percepción al color hace parte del tamizaje para determinar discromatopsias, de esta manera para el malabarista puede ser un factor importante ya que su rutina mezcla colores en contrastes que se resaltan en diversos momentos del día en donde la adaptación a la luz puede influenciar a la hora de realizar el acto ya que puede disminuir o aumentar la sensibilidad a los fotorreceptores y por ende la misma percepción del objeto en manipulación(14).

Tabla 1. Cuestionario para el diagnóstico de ojo seco de Donate

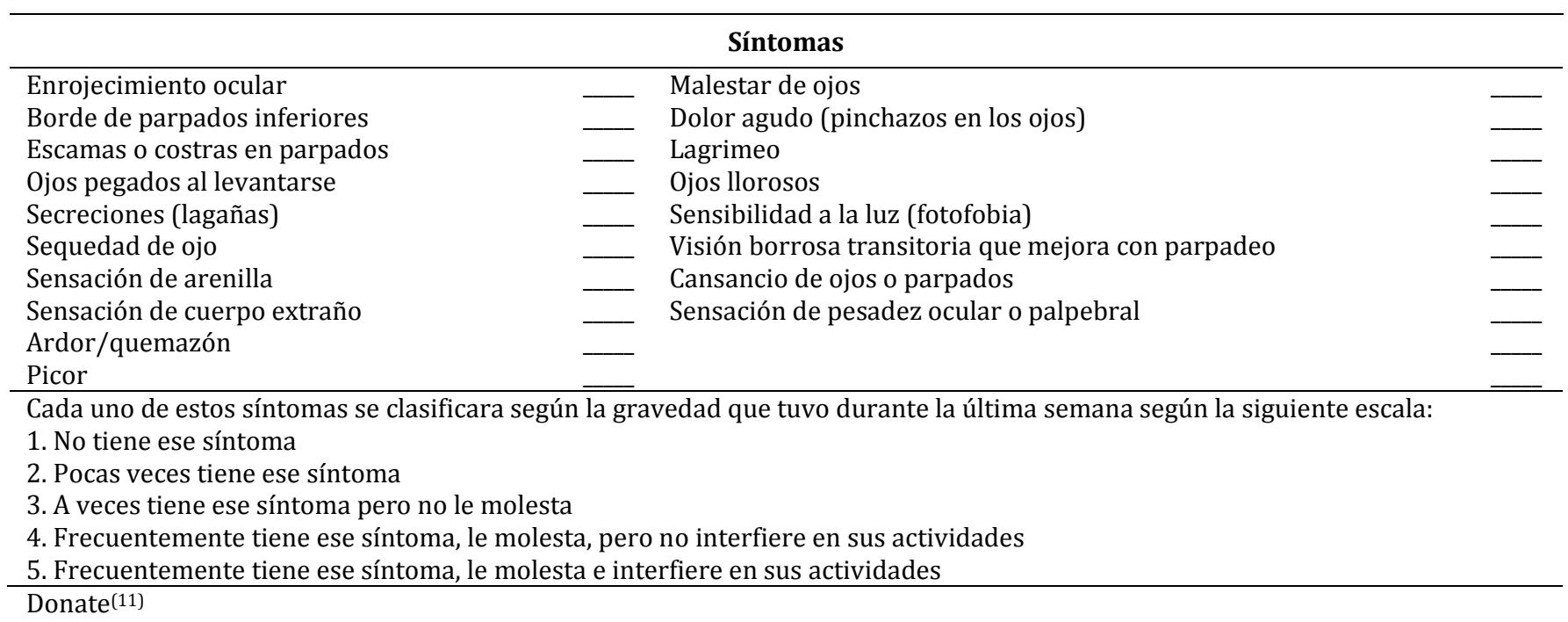

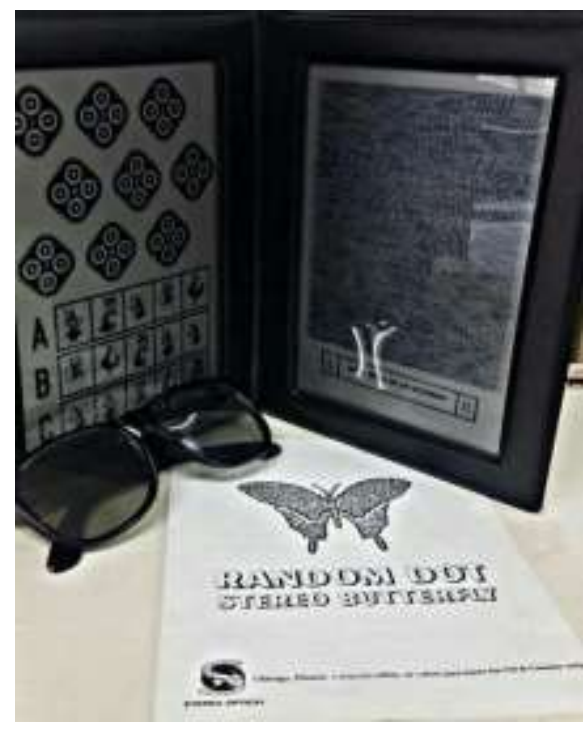

Figura 1. Test de Random Dot. Prueba para evaluar la estereopsis ${ }^{(15)}$

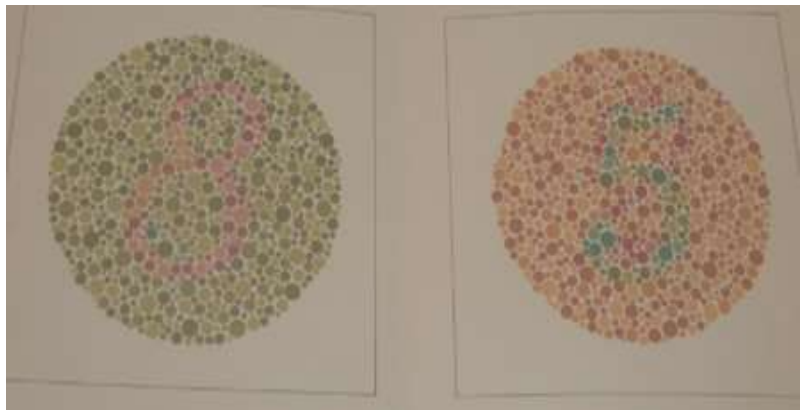

Figura 2. Test de Ishihara. Prueba de tamizaje que permite identificar alteraciones en la visión al color(13)

\section{Etapa final, campaña social}

Como fase final del trabajo se realizó una campaña utilizando herramientas del marketing social en salud, que facilitan la comprensión de la población acerca de los marcos culturales de comportamiento social en relación con el estado de salud de los individuos por medio de diversos medios de divulgación. La campaña social para esta investigación buscó sensibilizar a los actores 
sociales (transeúntes y conductores), sobre el desarrollo del malabarismo en la ciudad, involucrándose activamente y cambiando su perspectiva sobre el fenómeno del malabarismo y la importancia de la salud visual y ocular en el empleo informal de las calles. Se buscaron diferentes medios de divulgación tanto on-line como off-line(16). On-line se utilizó la página web: http://malabaristasparadi.wixsite.com/sociedad -malabarista, donde se dio a conocer la información general del proyecto (misión, visión, objetivos, fotografías y videos), los resultados encontrados en las pruebas visuales y oculares en los malabaristas participantes, a su vez la aplicación wix para la formación de la página web permitió el enlace directo a las redes sociales (Facebook) de cada uno de los perfiles del investigador, dinamizando y difundiendo dicha información a un público masivo. Desde la divulgación off-line se empleó un folleto informativo y la estrategia cara en el agujero (Face in Hole) utilizando una fotografía de un malabarista sin rostro, el rostro era reemplazado por el del transeúnte o conductor que deseaba conocer lo que se estaba promocionando. Para conocer el impacto de esta campaña se realizó una encuesta de satisfacción a cada participante, está se validó previamente realizando una prueba con un grupo de 50 personas con características semejantes a la población estudiada (Tabla 2).

Tabla 2. Herramienta de marketing utilizada en este estudio

\begin{tabular}{l}
\hline \multicolumn{1}{c}{ Encuesta de satisfacción } \\
\hline - ¿Es sencillo el acceso a la información? \\
- ¿La información se presenta de una forma dinámica? \\
- ¿Cree usted que esta campaña social logra transformar la \\
percepción que se tienen de los malabaristas? \\
- ¿La información que se brinda en las redes sociales es \\
clara y concisa? \\
- ¿Al acceder a este medio se cumplieron sus expectativas? \\
- ¿Recomendaría esta campaña a más personas? \\
\hline Responda las preguntas anteriores en base a la escala \\
presentada \\
1. Totalmente en desacuerdo \\
2. En desacuerdo \\
3. Ni totalmente de acuerdo ni en desacuerdo \\
4. De acuerdo \\
5.Totalmente de acuerdo \\
\hline
\end{tabular}

\section{Consideraciones éticas}

Se contó con el consentimiento informado escrito los participantes en cada fase del proyecto, así como para las validaciones de preguntas en las encuestas y entrevistas. De acuerdo con lo establecido en la resolución 8430 de 1990 y la declaración de Helsinki. El proyecto fue evaluado y avalado por el Comité de Ética de la Facultad de Ciencias de la Salud de la Universidad de La Salle.

\section{Resultados}

\section{Características socio-demográficas}

El estudio contó con la participación de 15 malabaristas, hombres (8) y mujeres (7); El promedio de edad en la población estudiada fue de 22 (DS \pm 2$)$ años. Todos los participantes de esta investigación eran estudiantes universitarios de carreras relacionadas a las áreas de las ciencias sociales: psicología (5), educación (4) y trabajo social (6). Todos laboraban en el arte del malabarismo en las localidades de Suba, Engativá y Teusaquillo de Bogotá.

De acuerdo con la clasificación del estado socioeconómico en Colombia, los estratos se gradúan de uno a seis, los malabaristas estudiados pertenecen al estrato dos a tres, donde se acogen a los usuarios con recursos económicos bajos(17). En cuanto al acceso a seguridad social solo es privilegiado uno de los participantes ya que es beneficiario por sus padres los otros demás sólo cuentan con una póliza de seguro contra accidentes, pero sólo los ampara dentro de la universidad. En general los participantes refirieron que las ganancias semanales dependen de diferentes factores, como el clima (lluvia, soleado), la clase de conductor (buena actitud, mala actitud), el tiempo de cambio de luz en el semáforo ( 30 a 45 segundos), con una ganancia aproximada de acuerdo al día entre $\$ 5.000$ a $\$ 60.000$ pesos colombianos.

\section{Diario de campo}

Para facilitar la descripción de lo observado se realizó un análisis de categorizando la observación realizada en el tiempo de práctica y 
por otro lado la observación en el tiempo laboral (semáforos). (Tabla 3)

Tabla 3. Análisis de contenido diario de campo

\begin{tabular}{cll}
\hline Objetivo & Categorización & \multicolumn{1}{c}{ Subcategorización } \\
\hline & $\begin{array}{l}\text { Observación: la } \\
\text { práctica }\end{array}$ & $\begin{array}{l}\text { Cultura universitaria } \\
\text { Escenarios lúdicos } \\
\text { Acto de autoformación }\end{array}$ \\
campo & $\begin{array}{l}\text { Acto final } \\
\text { Observación: } \\
\text { desde lo laboral }\end{array}$ & $\begin{array}{l}\text { Cultura del semáforo } \\
\text { Ambiente urbano }\end{array}$ \\
\hline $\begin{array}{l}\text { Codificación del diario de campo de acuerdo a los aspectos } \\
\text { observados dentro del mismo }\end{array}$ &
\end{tabular}

El tiempo de práctica se desarrolla en un entorno popular en las horas de la tarde donde se observa un gran número de universitarios realizando diversas actividades académicas y sociales que contribuyen a la construcción de una cultura universitaria. Este escenario posee espacios amplios y zonas verdes que cuentan con una iluminación natural donde se distingue con claridad los objetos y herramientas que hacen parte del escenario lúdico del malabarismo como se presenta en el siguiente apartado: "Allí el lugar posee una diversidad de herramientas como telares, que son colocados en árboles grandes de alta resistencia para que el individuo realice sus movimientos con mayor seguridad. Hay personas que amarran una cuerda a dos árboles convirtiéndose en una cuerda floja donde el practicante desarrolla equilibrio".

Este ambiente educativo muestra cómo el malabarista toma su tiempo de práctica como un acto de autoformación en el que aprende no solo a mejorar las habilidades frente al escenario sino también a manejar al público que lo contempla, como se observa en la siguiente cita: " $L a$ actividad del malabarismo da a lugar en varios momentos... alrededor de estos hay diversos espectadores que disfrutan del arte". Por otro lado, respecto al tiempo laboral la anotación se desenvuelve en un semáforo de la localidad de Suba en las horas de la tarde, donde transcurre una gran cantidad de transeúntes y conductores que reflejan un estado económico asequible, una posible ventaja para el malabarista como se referencia en la siguiente cita "...hay un gran número de transeúntes y conductores, se observa que son personas con una capacidad económica sostenible por las finas marcas de autos y estilos de ropa que poseen". Estos disponen un tiempo para participar en la actividad del malabarismo de forma indirecta, pues el tiempo del semáforo los localiza en la espera de cruzar las calles como se puede expresar en la siguiente anotación: “Los conductores al esperar el cambio del semáforo que tarda en promedio entre 30 a 45 segundos, aprovechan para interactuar visualmente con la presentación del malabarista, además allí convergen también las personas que pasan por las cebras peatonales".

El malabarista está listo en la cebra peatonal para comenzar su acto final y demostrar los resultados de su tiempo de práctica. Esto involucra la selección del instrumento de trabajo, un vestuario llamativo y una mayor exactitud a la hora de la manipulación, pues su objetivo es llamar la atención del observador y lograr un buen insumo económico por su trabajo: "Tanto conductores como transeúntes, en su inconsciente, califican la calidad y la atracción de cada acto, algunos conductores contribuyen a este empleo informal con algunas monedas o billetes". La actividad se desenvuelve en un día soleado con vientos frecuentes en un ambiente urbano bogotano, donde se observa contaminación tanto aérea como auditiva por lo carros que transitan; sin embargo, el malabarista manifiesta al concluir el día laboral la molestia ocular y corporal como se observa en el apartado: "Es una tarde soleada, nuestro participante toma un merecido descanso pues siente irritación ocular y dolor corporal".

\section{Entrevistas}

Como resultado de la codificación de primer nivel con la de segundo nivel, se encontró que los malabaristas se perciben a sí mismos como actores importantes para la cultura de la ciudad, además consideran que su actividad no solo obedece a una manera de obtener ingresos, sino que ante todo es un arte, ya que tiene una finalidad estética y comunicativa. Ellos expresan sus ideas, emociones, o la visión del mundo, utilizando recursos como aquellos derivados de lo plástico, lingüístico, sonoro, corporal y en la mayoría de las veces mixto. Además, consideran 
que al hacer parte de la cultura de la ciudad contribuyen a la trasmisión de ideas y valores. Un ejemplo de lo descrito por uno de los malabaristas que enmarca algunos de estos análisis es el siguiente "nosotros hacemos arte y con esto podemos vivir y ayudar a que la vida de la ciudad sea más amable".

Teniendo en cuenta las respuestas a las entrevistas realizadas a los conductores y transeúntes para conocer su percepción sobre el malabarismo, se encontró que la primera impresión para éstos actores sociales, es que los malabaristas son personas vagas o estudiantes de alguna ciencia social, además que realizan la actividad sólo por "pedir plata" como parte de la cultura del rebusque; otras personas los consideran viciosos anotando que son alcohólicos o drogadictos. Algunas personas aprecian los actos que ellos realizan y lo ven como algo divertido, y piensan que los malabaristas realizan esa actividad "como un pasatiempo".

Los malabaristas no perciben como importante la salud visual en su trabajo, ya que aunque se habían realizado exámenes visuales alguna vez en su vida y les habían prescrito corrección óptica, ellos decidían no utilizarla pues muchas veces se constituía, según ellos, en un peligro para el desarrollo de sus actos, además ellos consideran que utilizar cualquier ayuda visual es algo incómodo y anti estético, puesto que no combina con sus trajes de presentación como lo manifiesta uno de los participantes: "usar gafas o lentes de contacto es un estorbo para realizar adecuadamente mi acto, además no me veo bien".

\section{Estado ocular y visual}

Antes de iniciar el tamizaje visual se realizó un examen externo, el cual evaluó el grado de normalidad de los anexos oculares y el segmento anterior del ojo, descartando la presencia de patologías como una catarata o pterigión, que afectaran el desarrollo de los test visuales establecidos en la investigación. Se encontró que de los quince participantes sólo una persona contaba con corrección óptica prescrita hace dos años y su uso era de tipo ocasional, los otros participantes no se realizaban examen de optometría desde el ingreso a la universidad ( 2 a 3 años atrás).

Para conocer el estado ocular se aplicó el cuestionario de Donate. Al evaluar la sintomatología se tuvo en cuenta la frecuencia o número de veces que el sujeto presentaba la molestia ocular. En cada pregunta el participante clasificaba dicha frecuencia de 0 a 4 (Tabla 1), el $100 \%$ del participantes estudiados reportaron enrojecimiento ocular, sensación de arenilla, sensación de picor, sensación de pesadez ocular o palpebral; todos estos síntomas se presentaban frecuentemente, les causaba molestia y consideraban que interferían en sus actividades diarias. Al evaluar con pruebas de tamizaje aspectos de la función visual como: estereopsis y visión del color, se encontró que los malabaristas participantes de este estudio no presentaron alteraciones, obteniendo en la visión en profundidad 20" de arco, que es considerada normal y ninguna alteración respecto a la visión del color.

\section{Campaña social}

Como lema de la campaña se definió: "ponte en las "pelotas" de los demás", se buscó una frase fácil de recordar, que asociara algún elemento empleado por los malabaristas y a su vez se pensó que éste pudiera prestarse para otro tipo de interpretaciones, generando curiosidad y atracción por parte del público. Gracias a los medios de divulgación tipo on-line el público general, tuvo acceso a una página web donde se dio a conocer el grupo investigador "Paradigma", y la información relacionada con la investigación. La red social Facebook fue utilizada para dinamizar dicha información y compartirla con un público masivo. El impacto de la campaña fue evaluado por medio del número de comentarios de usuarios de las redes sociales que en este caso fueron siete; visualización del video que tuvo 45; número de "me gusta o likes" en Facebook que fueron ciento cuatro (104) y en la página web 39, todo esto durante una semana.

Desde la divulgación off-line utilizando la estrategia cara en el agujero (Face in Hole) se contó con la participación de 30 personas. Con la ayuda de un folleto se mostró la importancia de 
la salud visual y ocular en el desarrollo de esta actividad y se promocionó la página web para quien estuviera interesado. Finalmente, para conocer el impacto de esta campaña en su totalidad, se realizó una encuesta de satisfacción de seis preguntas a cada participante, empleando escala de Likert (Tabla 2). En las categorías de acuerdo/desacuerdo el $80 \%$ de los participantes tuvieron un efecto positivo respecto al impacto generado con la campaña.

Con la información obtenida en las etapas mencionadas en la metodología, se pueden sintetizar los resultados de la fase cualitativa en las siguientes categorías y subcategorías de acuerdo con cada objetivo (Tabla 4)

Tabla 4. Análisis por categorías y subcategorías

\begin{tabular}{cll}
\hline Objetivo & Categorización & Subcategorización \\
\hline General & $\begin{array}{l}\text { Importancia salud } \\
\text { visual y ocular }\end{array}$ & $\begin{array}{l}\text { Impedimento } \\
\text { Sin importancia } \\
\text { Riesgo en el trabajo }\end{array}$ \\
& Percepción de los & Artistas urbanos \\
& malabaristas en sí & Cultura de la ciudad \\
mismos & Subsistencia \\
& Percepción de la & Vagos (drogadicto) \\
& sociedad hacia los & Estudiantes \\
& malabaristas & Rebusque \\
Específico & Campaña social & Visible \\
& & Accesible \\
& & De Impacto \\
\hline
\end{tabular}

Clasificación por organización conceptual de acuerdo a cada objetivo establecido en la investigación

\section{Discusión}

El arte es la manera como el ser humano expresa e interpreta de manera estética y comunicativa su visión del mundo. Las artes urbanas nacen como sistemas dinámicos, que están en constante cambio, como resultado de la realidad que cada individuo percibe, originando movimientos culturales que nacen fuera de los circuitos institucionales y que pueden aparecer como reflejo de los nuevos conflictos o tensiones sociales(5). El malabarismo es un ejemplo claro de aquel movimiento cultural que ha crecido en las calles de Bogotá en las últimas décadas, convirtiéndose en un arte diferencial y libre que contribuye con la transmisión de ideas y valores indispensables para la cultura ciudadana, como lo han referido los participantes de este estudio. A su vez, esta actividad provoca una invisibilización de la dimensión laboral de las prácticas artísticas asentando representaciones sociales dicotómicas acerca del arte y el trabajo(18).

Por lo anterior, surge el concepto del rebusque como una estrategia cultural que destaca el aumento de diferentes factores sociales como el incremento del empleo informal. Según la Organización Internacional del Trabajo (OIT) el $50 \%$ de este tipo de empleo pertenece a las ciudades de los países que se encuentran de bajos a medianos ingresos, como lo es Colombia(19). De allí resulta la idea de que el empleo informal, lo constituye una parte de la comunidad denominada "la sociedad de semáforo". Usando el espacio público como escenario, esta diversidad de personajes heterogéneos (vendedores ambulantes, cuenteros, mendigos, entre otros) abre paso a la funcionalidad de diversos discursos, a partir de múltiples percepciones e infinitas prácticas que ejercen sus habitantes en la espera de un cambio de color de rojo a verde.

En efecto, la ciudad actúa como escenario y como demanda productora de estas prácticas, siendo los malabares los más destacados de tipo artístico(20). Como resultado de las entrevistas realizadas en este estudio, los malabaristas participantes demuestran que la ejecución de esta actividad en las calles de la ciudad es la manera como ellos "obtienen ingresos diarios" siendo parte de esta cultura del "rebusque", la cual busca suplir sus necesidades básicas para subsistir. Esta idea se enfrenta con el punto de vista de algunos espectadores, pues ellos piensan que esta actividad sólo la realizan para "pedir plata", y lo asocian con estereotipos de personas vagas (gente sin oficio) o viciosas, anotando que el dinero que obtienen sólo es para invertir en alcohol o drogas. No obstante, hay que entender que buscar empleo formal desarrollando esta actividad, no es fácil ni común en Bogotá. Por esto, algunos malabaristas urbanos estudian carreras universitarias ${ }^{(21)}$ que les permiten tener ingresos fijos en el futuro y no depender completamente del malabarismo. 
Todos los participantes de esta investigación estudiaban carreras relacionadas a las áreas de las ciencias sociales como: psicología, educación y trabajo social y desde el punto de vista de las bellas artes, las artes pláticas. Podría pensarse que estas carreras les permiten comprender el comportamiento de los seres humanos, al ser parte de un fenómeno como es el malabarismo considerándose como manifestación simbólica social, por ello lo practican(22).

Por otra parte, el acceso de los malabaristas al Sistema de Seguridad Social (SSS) se convierte en una problemática ya que los parámetros para la formalización y la generación de este empleo informal por subsistencia no se encuentran reglamentados directamente por la constitución política, y su disposición refiere que el trabajo informal en general no alcanza a entrar en la categoría de contratos, ni siquiera de tipo ocasional o transitorio (Decreto 2616 de 2013)(23). De esta manera, los trabajadores informales, aun prestando personalmente sus servicios en una relación subordinada y remunerada, generalmente son excluidos de la afiliación a los sistemas de pensiones, riesgos laborales y subsidio familiar, lo que implica su desprotección en materia de seguridad social(22). En el estudio desarrollado, sólo uno de los participantes contaba con acceso a la seguridad social por ser beneficiario de los aportes de sus padres y los demás no contaban con esta afiliación, siendo excluidos de la protección dada por el estado a trabajadores formales $\mathrm{e}$ independientes.

Posiblemente por esta razón los participantes de este estudio no ven significativo el compromiso de la salud visual y ocular en el desarrollo de esta actividad, pues si para ellos tener acceso a servicios de medicina general resulta complicado, el hecho de asistir a otros servicios particulares implica gastos adicionales que ellos evitan tener, a no ser que sean totalmente necesarios. Los participantes de esta investigación no habían asistido a una consulta de optometría un buen tiempo atrás. Ellos refirieron que en esa ocasión les prescribieron "gafas" que no consideraban importantes, además anotaron que desconocían que debían realizarse una evaluación optométrica anualmente, para prevenir enfermedades a largo plazo que dificultaran la realización de ciertas actividades rutinarias y el desempeño de su trabajo informal(24).

No obstante, según Bohórquez y Pérez, se encontró que la radiación ultravioleta en Bogotá supera ampliamente el valor de 11 de acuerdo a la escala dada por la Organización Mundial de la Salud y la Comisión Internacional de Iluminación (CIE), indicando que ese valor corresponde a un índice extremadamente alto, llegando a producir daños biológico en piel y ojos en corto tiempo de exposición(25). Teniendo en cuenta que los malabaristas trabajan más de 8 horas en un semáforo así sus actos duren menos de 30 segundos, estarían extremadamente expuestos a dicha radiación, haciéndolos susceptibles a generar cataratas, pterigión e incluso cáncer ocular, patologías que de forma monocular o binocular afectan al sistema visual generando disminución de la agudeza visual, la estereopsis, visión del color, entre otros(26).

La Secretaría Distrital de Ambiente de Bogotá reportó concentraciones de monóxido de carbono superiores a los niveles máximos permitidos nacionales, debido a esto Bogotá se conoce a nivel Latinoamérica como una de las ciudades con mayor contaminación atmosférica; a su vez la calidad de aire de la ciudad se ve afectado por el material particulado respirable $\left(\mathrm{PM}_{10}\right)$, generando cronicidad en el organismo con aumento de enfermedades vasculares y pulmonares(27). A su vez, de acuerdo con Herrera et al.(28), el contacto con la contaminación ambiental puede ocasionar enfermedades como ojo seco, el cual involucra síntomas tempranos como irritación ocular, disminución de la calidad y cantidad de película lagrimal e incidencia de infecciones en los párpados por falta de higiene.

Lo anterior puede estar relacionado con los síntomas que refieren los participantes de la investigación en el cuestionario de síntomas de Donate, en el cual el 100\% de la población refirió enrojecimiento ocular y la sensación de arenilla, manifestando mayor frecuencia cuando llevan mucho tiempo de exposición al sol, hecho que 
puede indicar que estos pacientes tienen una mayor predisposición a tener un ojo seco en un futuro.

Según el reporte otorgado por la Secretaría Distrital de Salud, en diciembre del 2010 se encontró que en los trabajadores informales el sistema visual se ve afectado en un $18 \%$, siendo la disminución de visión el síntoma más destacado ocupando un 33\%(29). Los malabaristas participantes de este estudio no presentaron evidencia de padecer algún trastorno en la función visual (visión de los colores y visión de profundidad), quizás por el tiempo que lleven desempeñando la actividad, las horas que lo hacen y practican o por la edad, ya que era jóvenes y su sistema visual aún no ha sufrido proceso de envejecimiento(30). Por esto quizás, no veían la importancia de la salud visual en su trabajo ya que sus actos los realizaban con precisión. Igualmente, consideraban que el uso de cualquier elemento para mejorar su visión (ej.: "gafas" o lentes de contacto) podría convertirse en algo antiestético, no armónico con su indumentaria de presentación y que incomodaría el desarrollo de su actividad. Incluso sería un riesgo para ellos, por ejemplo, si en sus actos utilizaran fuego o cualquier otra sustancia o elemento, porque en el caso de usar lentes de contacto podría producir irritación ocular(31) o al usar "gafas" generar empañamiento. Sin embargo, esto es controvertible, pues según Jiménez y Perdomo, las gafas de protección son ideales para el desarrollo de diferentes trabajos ya que como su nombre lo indica permiten la protección en este caso ocular, combinando materiales que brindan la protección a la radiación ultravioleta, la resistencia a impacto junto con protección lateral para evitar el salpique de químicos en los ojos y la incidencia de agentes contaminantes en el lugar de trabajo(32).

En el mercado se encuentran diferentes diseños que proveen ventilación para evitar que se genere empañamiento y por lo tanto los lentes sean efectivos ópticamente(33). El gobierno local en Bogotá ha dispuesto desde 2010, como parte de la prevención y protección a la salud de los trabajadores informales estrategias que buscan mejorar la condición laboral. Sin embargo, identificaron que una de las barreras de acceso se debe a que el $5,1 \%$ de la población no cree en la efectividad de los servicios de salud otorgados por el gobierno, por ende, no se hacen partícipes ${ }^{(29)}$.

Finalmente, se realizó una campaña social, que generó curiosidad por parte de conductores y transeúntes, quizás porque el título "ponte en las "pelotas" de los demás", que daba quizás un doble sentido a la misma, constituyéndose en una estrategia publicitaria muy utilizada para producir "morbo", fenómeno particular en donde el ser humano tiene una atracción por aquella construcción discursiva que hace de cada noticia una aproximación a lo íntimo (sexual), los dolores ajenos, la pobreza(34). En la actualidad la publicidad utiliza medios de divulgación tipo offline y online, los cuales facilitan el acceso de los espectadores a diversos temas. En el caso del medio tipo online el acceso a internet ha impuesto cambios importantes en los esquemas comunicativos que ponen en contacto de manera inmediata a emisores y receptores en todo el mundo. Por esta versatilidad, el usuario tiene la libertad de escoger y ampliar su conocimiento mediante el alcance de la información que es brindado por diversas páginas web o redes sociales. Al menos el $90 \%$ de los usuarios de internet conoce hasta cuatro redes sociales y un $70 \%$ hace parte de una aproximadamente( ${ }^{(35)}$.

Por lo anterior, el medio de divulgación tipo online empleado en esta investigación, fue exitoso, dado que logró la atención de usuarios de redes sociales. Esto se evidenció en el número de visualizaciones, comentarios y me gusta de la campaña on-line durante una semana. La estrategia publicitaria offline, ha perdido auge debido a la llegada del tipo online, aunque se sigue sosteniendo como un instrumento publicitario indispensable para mostrar ideas de forma abierta y exitosa(35). Por esto, se decidió incluir como parte de la campaña, la actividad "Face in Hole" porque al existir una participación activa en el desarrollo de este esquema( ${ }^{36)}$ el actor social pudo ponerse en el lugar del malabarista y en conjunto con el aporte del folleto entregado a cada participante que incluía 
el vínculo a la página web, video en YouTube y un correo de contacto, se logró que el actor social tuviera su propio criterio acerca de cómo se ve el malabarista en la sociedad y la importancia de la salud visual y ocular en su labor.

\section{Conclusiones}

La salud visual y ocular no tiene gran importancia para los malabaristas participantes del estudio ya que el uso de corrección óptica es concebido como algo antiestético y peligroso. Sin embargo, se observó que los malabaristas participantes presentaban síntomas oculares como enrojecimiento ocular y que estaban expuestos a factores de riesgo visuales que podían impactar negativamente el desempeño y calidad de sus actos circenses.

Los malabaristas se perciben como actores sociales que por medio de su arte contribuyen a la construcción de una sociedad educada, mientras que para el común de la gente esto es simplemente un trabajo informal de "rebusque" que fomenta el consumo de alcohol $y$ drogadicción entre los que lo practican.

La campaña social diseñada para el estudio logró sensibilizar a transeúntes, conductores y público en general frente a este tipo de actividades que ocurren en las calles y permitieron el reconocimiento sobre la existencia del otro. Además, permitió que los actores sociales y el mismo malabarista reconocieran la importancia de la salud visual y ocular durante el desarrollo de su trabajo informal.

\section{Limitaciones y fortalezas}

A pesar de que se logró que un número significativo de malabaristas participaran, muchos de los malabaristas no lo hicieron, por cuanto no disponen su arte sin conseguir una retribución a cambio.

\section{Conflicto de intereses}

No existen conflictos de intereses.

\section{Referencias}

1. Malaver C. El arte de los malabares; entre la necesidad y el arte [Internet]. Bogotá: El tiempo; 2010. Disponible en:

http://www.eltiempo.com/archivo/documento/CMS7023407

2. Penton N. IJA 2015 Sneak Peek. International Jugglers' Association. 2014. p. 1-10.

3. Mendoza R. Pelicula "La Sociedad del Semáforo" [Internet]. Colombia: Cine Colombia; 2010. Disponible en:

http://www.proimagenescolombia.com/secciones/cin e_colombiano/peliculas_colombianas/pelicula_plantilla .php?id_pelicula $=1762$

4. Webb JW, Bruton GD, Tihanyi L, Ireland RD. Research on entrepreneurship in the informal economy: Framing a research agenda. J Bus Ventur. 2013;28(5):598-614.

5. Rojas A. Malabarismo: más que un arte, una forma de vida. Bogotá: Paraíso cultural. 2013.

6. Departamento Administrativo Nacional de Estadística. Gran Encuesta Integrada de Hogares. Resumen de Encuesta. Bogotá: DANE; 2016.

7. Vega MM, Martínez KA. Estudio empírico de calidad de vida laboral, cuatro indicadores: satisfacción laboral, condiciones y medioambiente del trabajo, organización e indicador global, sectores privado y público. Desarrollo, aplicación y validación del instrumento. Horizontes Empres. 2015;26;8(1):23-50.

8. Franco JF, Contreras JF. Exposición de peatones a la contaminación del aire en vías con alto tráfico vehicular. Rev Salud Pública. 2016;18:179.

9. Gale NK, Heath G, Cameron E, Rashid RS. Using the framework method for the analysis of qualitative data in multi-disciplinary health research. BMC Med Res Methodol [Internet]. 2013;13:117. Disponible en: https://doi.org/10.1186/1471-2288-13-117

10. Hernández RM. La investigación cualitativa a través de entrevistas: su análisis mediante la teoría fundamentada. Cuest Pedagógicas. 2014;23:187-210.

11. Donate J, Benítez del Castillo JM, Fernández C, García J. Validación cuestionario para diagnóstico de ojo seco. Arch Soc Esp Oftalmol. 2002;77(9):493-500.

12. De La Cruz A, Morale SE, Jost RM, Kelly KR, Birch EE. Modified Test Protocol Improves Sensitivity of the Stereo Fly Test. Am Orthopt J. 2016;66:122-5.

13. Pouw A, Karanjia R, Sadun A. A method for identifying color vision deficiency malingering. Graefe's Arch Clin Exp Ophthalmol. 2017;255(3):613-18.

14. Bermúdez ML, Camacho M, Figueroa-Olarte LF, Medrano SM León A. Relación entre la ambliopía y el proceso de lectura [Internet]. Rev Cienc y Tecnol para la Salud Vis y Ocul. 2016;14(2):83-91. Disponible en: http://revistas.lasalle.edu.co/index.php/sv/article/vie w/3835

15. Hibbard PB, Scott-Brown KC, Haigh EC, Adrain M. Depth perception not found in human observers for static or dynamic anti-correlated random dot stereograms. Plose one [Internet]. 2014;9(1):e84087. 
Disponible

en:

https://doi.org/10.1371/journal.pone.0084087

16. Lorente A, Fernández L. La eficacia de la integración offline y online en la estrategia de comunicación corporativa. Gandía: Universidad politécnica de Valencia; 2013.

17. Corte Constitucional de Colombia. Ley 142. [Internet]. Colombia: Corte Constitucional; 1994. Disponible en: www.corteconstitucional.gov.co/RELATORIA/2013/C263-13.htm

18. Montenegro-Vivero S. Escenarios callejeros un espacio de formación, exhibición y vivienda de artistas callejeros. [Internet]. Ecuador: Pontificia Universidad Católica del Ecuador; 2014. Disponible en: http://repositorio.puce.edu.ec/handle/22000/11752

19. Rodriguez GI, Calderon MA. La economía informal y el desempleo: el caso de la ciudad de Bucaramanga. Innovar. 2015;25(55):41-58.

20. Tejerina E. La sociedad del semáforo: los malabares y las diferentes prácticas sociales que confluyen en él. En: Encuentro panamericano de comunicación; Córdoba 2013. Córdoba: Universidad Nacional de Córdoba; 2013.

21. Acosta E, Barrantes N, Guerra-Muriel W, MaldonadoPalacios D, Silva-Lurduy J, F. AS. Apropiación y significación cultural y artística del espacio urbano: el caso del chorro de Quevedo en Bogotá. Aposta. 2016;(71):62-94.

22. Hernández-Bello A, Gutiérrez-Bonilla ML. Vulnerabilidad y exclusión en salud: datos y relatos de la situación de la población desplazada en Bogotá. Bogotá: Pontificia Universidad Javeriana; 2015.

23. Ministerio del Trabajo. Decreto 2616 el 2013. Bogotá: MinTrabajo; 2013.

24. American Academy of Ophthalmology. Get Screened at 40 [Internet]. EEUU: American Academy of Ophthalmology; $2015 . \quad$ Disponible en: https://www.aao.org/eye-health/tipsprevention/screening

25. Pérez JF, Bohórquez J. Análisis correlacional entre la radiación ultravioleta del sol y la prevalencia de conjuntivitis actínicas en escolares entre 5 y 12 años de edad, a tres alturas sobre el nivel del mar en el departamento de Cundinamarca. Cienc y Tecnol para la Salud Vis y Ocul. 2007;(9):35-42.

26. Legrá RJ., Ortega YM, Rodríguez DD, Cantillo RE, Borroto YJ. Caracterización clínico-epidemiológica de pterigium. Rev Inf Científica. 2017;96:355-62.

27. Alcaldía Mayor de Bogotá DC. Documento Técnico Línea de Intervención Aire, Ruido y Radiación Electromagnética 2011- 2023. Bogotá: Alcaldía Mayor de Bogotá; 2011.

28. Vicente-Herrero MT, Ramírez-Iñiguez de la Torre MV, Terradillos-García MJ, López-González AA. Síndrome del ojo seco. Factores de riesgo laboral, valoración y prevención. Semer Fam. 2014;40(2):97-103.

29. García J, Suárez N, Tovar MT. La estrategia de entornos de trabajo saludables en Bogotá: evaluación y propuestas de ajuste -2011. Rev la Fac Med. 2013;61(1):S1.
30. Perez N, García L, Montero BM, Cruz A. Valoración del paciente geriátrico. Med Form Médica Contin Acreditado. 2014;(62): 3641-3658.

31. Schweizer H, Perez I. Contact Lens Induced Ocular Surface Alterations. In: Martin R, Corrales R, editors. Ocular Surface: Anatomy and Physiology, Disorders and Therapeutic Care. Boca Raton: Taylor \& Francis Group; 2013. p. 257-87.

32. Jiménez I, Perdomo C. Protección Ocular y Facial. En: La Salud ocupacional en Optometría. Bogotá: Universidad de la Salle; 2009. p. 135-151.

33. 3M Productos de Protección Personal. Soluciones de protección ocular. [Internet]. España: 3M; 2014. Disponible en: http://solutions.productos3m.es/3MContentRetrieval API/BlobServlet?lmd=1395761627000\&locale $=$ es_ES \& assetType $=$ MMM_Image $\&$ assetId $=1361792225833 \& b l$ obAttribute=ImageFile

34. Paz-Pirela JV, Paz-Pirela JA. Marketing Social Como Estrategia Para Fomentar La Responsabilidad Social En Las Televisoras De La Costa Oriental Del Lago//Social Marketing As A Strategy To Promote Social Responsibility In The Television Stations of The Eastern Coast Of Lake. Cicag. 2014;12:160-77.

35. Miniwatts Marketing Group. Estadísticas de volumen de usuarios de Facebook y Redes Sociales en el mundo. España: Internet World Stats. 2015.

36. Schneider JP. Morbo Una aproximación al sensacionalismo televisivo. Buenos Aires: Universidad de Buenos Aires; 2015. 